\title{
Unimanual to Bimanual: Tracking the Development of Handedness from 6 to 24 Months
}

\author{
Eliza L. Nelson ${ }^{1}{ }^{*}$, Julie M. Campbell ${ }^{2}$, and George F. Michel ${ }^{1,2}$ \\ ${ }^{1}$ Center for Developmental Science, University of North Carolina at Chapel Hill, Chapel Hill, NC, \\ United States \\ 2Department of Psychology, University of North Carolina at Greensboro, Greensboro, NC, United \\ States
}

\section{Abstract}

Manual skills change dramatically over the first two years of life, creating an interesting challenge for researchers studying the development of handedness. A vast body of work to date has focused on unimanual skills during the period from the onset of reaching to walking. The current study sought to connect such early unimanual hand use to later role-differentiated bimanual manipulation (RDBM), in which one hand stabilizes the object for the other hand's action. We examined hand use in 38 children over 16 monthly visits using a validated measure for assessing hand preference for acquiring objects when children were 6 to 14 months old. We also developed a new measure for assessing RDBM preference presented when children were 18 to 24 months old. The new measure reliably elicited RDBM actions in both toddlers and an adult control group $(N=15)$. Results revealed that some children show preferences for acquiring objects as infants; these preferences are stable and persist into their second year as new skills appear. Moreover, children with no hand preference during infancy shifted to left or right lateralized hand use as toddlers. Despite a higher incidence of left-handedness compared to adult norms, the majority of children were right-handed by 2 years of age.

\section{Keywords}

handedness; hand preference; infant; toddler; bimanual; manipulation

\section{Introduction}

Manual skills change dramatically over the first two years of life, making it particularly challenging to measure handedness longitudinally. Perhaps as a consequence, many researchers have focused their efforts either on a single skilled behavior involving the hands or a specific time point in development. A large literature has examined unimanual hand use for reaching to objects, and this work has been centered on the period from the onset of reaching through the onset of walking (e.g., Carlson \& Harris, 1985; Corbetta \& Thelen, 1999; Fagard, Spelke \& von Hofsten, 2009; Ferre, Babik \& Michel, 2010; Hinojosa, Sheu \&

\footnotetext{
(c) 2013 Elsevier Inc. All rights reserved.

*Corresponding Author: Florida International University, Department of Psychology, 25611200 S.W. 8th Street, Miami, FL 33199, Phone: (305) 348-4894, Fax: (305) 348-3879, eliza.nelson@ fiu.edu.

Publisher's Disclaimer: This is a PDF file of an unedited manuscript that has been accepted for publication. As a service to our customers we are providing this early version of the manuscript. The manuscript will undergo copyediting, typesetting, and review of the resulting proof before it is published in its final citable form. Please note that during the production process errors may be discovered which could affect the content, and all legal disclaimers that apply to the journal pertain.
} 
Michel, 2003; Michel, Ovrut \& Harkins, 1985; Michel, Tyler, Ferre \& Sheu, 2006; Ramsay, 1980; Rönnqvist \& Domellöf, 2006). Collectively, these studies have identified an early right hand-use preference at the group level amidst some variability in the trajectories of individual infants.

Much less well studied is bimanual hand use (for a recent review, see Greaves, Imms, Krumlinde-Sundholm, Dodd \& Eliasson, 2012). Broadly, symmetrical bimanual actions precede asymmetrical ones in development (e.g., Fagard \& Jacquet, 1989). Asymmetric bimanual actions are of greater interest because they involve the hands playing complementary and distinct roles (i.e., one hand holds the object for the other hand's actions). This type of advanced manual skill has been termed role-differentiated bimanual manipulation (RDBM). The ability to successfully reach for and grasp objects (approximately 4 months of age; see Berthier \& Keen, 2006) is temporally linked to the earliest observations of RDBM. Although rudimentary, Rochat (1989) described 4-monthold infants holding a toy in one hand and exploring its properties with the fingers of the opposite hand.

Although infants exhibit RDBM early in their first year, it is only a minor aspect of their repertoire. For example, Kimmerle, Mick and Michel (1995) found that RDBM accounted for less than $10 \%$ of all observed manual actions in 7 -month-olds. It is likely that the low rate of RDBM reported in young infants is driven by the affordances or properties of the objects. Perhaps not surprisingly then, hand-use preferences for RDBM only begin to appear during the 11 to 13 month age period (Kimmerle, Ferre, Kotwica \& Michel, 2010; Kimmerle et al., 1995; Michel, Ovrut, \& Harkins, 1985) when the infant begins coordinating RDBM actions.

RDBM skills continue to be refined through the second year of life as bimanual strategies shift from partially to fully differentiated hand use (e.g., Fagard, 1998; Fagard \& Jacquet, 1989; Fagard \& Marks, 2000; Fagard \& Pezé, 1997; Ramsay, Campos \& Fenson, 1979). For instance, Ramsay and Weber (1986) reported that only 50\% of bimanual actions in 12- to 13-month-old infants were completely differentiated, but by 17 to 19 months of age, that figure had increased to 78\%. More recently, Fagard and Lockman (2005) found that $64 \%$ of infants 6 to 12 months of age were successful in using both hands on a task requiring holding a container with one hand and pulling out a tube with the opposite hand. By 18 months of age, $100 \%$ of children used a fully differentiated strategy on the "tube/container" task. Tasks such as the "tube/container" that effectively constrain hand use are superior for measuring bimanual handedness as compared to tasks that do not clearly differentiate the roles of the manipulating hand and the stabilizing hand or could potentially be performed with one hand instead of two (e.g., Fagard \& Lockman, 2005; Fagard \& Marks, 2000; Geerts, Einspieler, Dibiasi, Garzarolli \& Bos, 2003). As in studies of unimanual hand preferences, studies of bimanual preferences have also found right-handedness (as measured by the manipulating hand) to be the group-level pattern. However, many of the existing studies in the literature have calculated bimanual preferences from a single task (e.g., Fagard \& Lockman, 2005) or fewer than 10 trials (e.g., Fagard \& Marks, 2000).

Building on previous research, our first aim of the current study was to develop a battery of tasks that reliably elicit RDBM actions. The objectives were twofold: (1) to sufficiently constrain hand use such that two hands were required to perform the given task, thus ensuring the roles of each hand were clearly defined and (2) to include a variety of actions to adequately assess hand use preference where the minimum number of data points was 20 . We selected 18 months as the starting target age as this is a time point at which the majority of children should be capable of performing completely differentiated bimanual actions. We expected that some actions such as unscrewing a lid would be more difficult than other 
actions. By providing such challenging items, we hoped to maintain children's interest in the toys throughout each test session as well as across test sessions in our longitudinal design. We also anticipated that by 24 months, children would be able to successfully complete all of the target RDBM actions.

Our second aim was to connect hand use preferences from the new RDBM battery to prior unimanual hand use data collected when the same children had been observed as infants over the 6 to 14 month period. During this time, both unimanual actions (manipulation distinct from the unimanual acquisition of objects) and RDBM actions comprise very small portions of the infant's manual repertoire and both exhibit hand-use preferences only in the later months of that period (Hinojosa et al., 2003; Kimmerle et al., 2010). Thus, the action of acquiring objects is the only manual action that remains constant through this developmental range that can serve as the basis for measuring hand-use preferences. Few studies have examined both unimanual and bimanual hand use in developing children. Previous research has revealed a relationship between hand-use preferences in these two domains of manual skill measured concurrently at various ages in development (e.g., Fagard, 1998; Fagard \& Lockman, 2005; Fagard \& Marks, 2000; Michel, et al., 1985). Using a cross-sectional design, Michel and colleagues (1985) reported that handedness for RDBM was concordant with handedness for unimanual manipulation (but not reaching) during the last months of the infant's first year. In a longitudinal study by Ramsay (1980), bimanual handedness measured at 13 months corresponded to unimanual handedness measured at 7 or 9 months in 23 of 28 infants.

The major limitation of these previous studies, whether cross-sectional or longitudinal, has been the assessment of the target manual skill. The current study is the first longitudinal attempt to connect unimanual and bimanual preferences, and emerging handedness patterns, over repeated monthly assessments in a large group of developing children $(N=38)$. We first assessed unimanual hand-use preferences for acquiring objects from 6 to 14 months ( 9 visits) using a validated infant measure developed for this age range (Michel et al., 1985). Next, we assessed bimanual hand use preferences for RDBM actions when children were 18 to 24 months (7 visits) using the new toddler measure that was designed to capture the advanced coordinated bimanual skills exhibited in the latter half of the second year of life. Finally, we administered the toddler measure to a control group of adults $(N=15)$ to confirm that the tasks chosen to assess bimanual handedness in a developing population were in fact effective in eliciting role-differentiated actions in individuals with established hand use preferences.

\section{Materials and methods}

\subsection{Participants}

2.1.1 Children-Thirty-eight children ( 21 females) participated in a longitudinal study investigating hand use at monthly intervals from 6 to 14 months (infant visits) and again from 18 to 24 months (toddler visits). Families were initially recruited for the infant portion of the project when their child was 5 months old using birth records obtained from the Guilford County Court House in North Carolina. Inclusion criteria included full-term pregnancy of at least 37 weeks gestation and delivery without complications. Sample families were representative of the ethnic backgrounds found in the local community $($ sample $=65 \%$ Caucasian White, 15.8\% African American, 13.2\% Multiracial, 2.6\% Hispanic, 2.6\% Other Race).

Data collection began at 6 months and each monthly assessment occurred within 7 days of the child's birthday. Children that had missed no more than 1 infant test session were recruited for the toddler visits. Three children missed 1 infant session, and three different 
children missed 1 toddler session. In sum, six children missed only 1 visit out of 16 over the course of the study. Three additional children (two males and one female) began the toddler portion of the project but missed 2 or more sessions. Their data were not included in the analyses.

2.1.2 Adults-Fifteen adults ( 11 females, $M \pm \mathrm{SD}=31 \pm 11$ years) also participated in the study to determine whether the items designed for toddlers reliably elicited roledifferentiated bimanual responses in adults. We chose parents or family members of children who were enrolled in the infant segment of our ongoing project. Adults were tested individually while the infant and any other family members were in a separate room. Thus, infants were not exposed to the contents of the toddler battery prior to their eligibility for that portion of our project and the adult data collected to validate the toddler battery was not connected to the child data reported in this study.

\subsection{Procedure}

All test sessions were conducted at the University of North Carolina at Greensboro Infant Development Center. The UNCG Institutional Review Board approved all of the procedures and parents gave written consent for their child to participate in this study. Parents received a $\$ 10$ Target GiftCard ${ }^{\circledR}$ for each lab visit. Test sessions were recorded with two Panasonic digital cameras linked by a Videonics mixer, providing overhead and left facing views of the child's actions combined into a single frame for later coding. Video coding was done offline with the Observer ${ }^{\circledR}$ XT program (Noldus Information Technology, v10). Reliabilities were calculated using percent agreement between two coders for each object presentation (up to 34 coding decisions per session), with coders scoring 7 to 8 videos from each age tested, or approximately $20 \%$ of the data.

2.2.1 Infant Handedness-Nine infant visits occurring monthly from 6 to 14 months of age assessed hand use for apprehending various objects using the validated infant handedness measure developed by Michel et al. (1985). Briefly, infants were seated on a parent's lap at navel height at a table and presented with 34 toys. Of these, 24 were presented singly at the infant's midline and the remaining 10 items were pairs of toys presented dually in line with the infant's shoulders. Infants were encouraged to reach for and manipulate the objects. Hand preference was scored offline from videotape as the hand used to acquire each toy (see Ferre et al. 2010 for additional details on this procedure; note: the sample reported here was born in 2009-2010 and therefore not included in the Ferre et al. 2010 sample). Reliability for the infant handedness measure was $93 \%$.

2.2.2 Toddler Handedness-Seven toddler visits occurring monthly from 18 to 24 months of age assessed hand use for role-differentiated bimanual manipulation (RDBM) using the new test battery. Presenters were blind to toddlers' infant hand preference status. As in the infant visits, toddlers were seated on a parent's lap at navel height at a table. Test objects were then presented one at a time at the child's midline. All objects were designed to require the use of the two hands together in an asymmetrical fashion such that the supporting hand stabilized the object for the opposing hand's manipulation and were difficult, if not impossible, to perform with a single hand. The objects were chosen to elicit target RDBM actions such as removing a toy from inside of another toy, unlatching a container, peeling a sticker, and unzipping a bag (Figure 1). Testing consisted of seven objects presented twice non-consecutively with three of those objects requiring multiple actions and seven objects presented once (Table 1). For objects with a series of actions, each step in the action was considered a separate data point. In total, there were 29 data points possible per session. Hand preference was scored offline from videotape as the manipulating hand in the RDBM action. All unimanual or unsuccessful bimanual attempts to complete the target action were 
discounted. Coders for the toddler data were blind to infant handedness status. Reliability for the toddler handedness measure was $96 \%$.

2.2.3 Adult Handedness-Adults were verbally asked to perform the target action (e.g., remove the ball from the tube), but were not given any instructions regarding how to do so (i.e., which hand(s) to use). Responses were scored in real-time by two independent observers. Reliability for the adult handedness measure based on percentage agreement across all observations was $98 \%$.

\section{Results}

\subsection{The new test battery reliably elicits RDBM actions in toddlers and adults}

Table 2 indicates the number of children sampled at each of the 7 toddler time points and the mean number of RDBM actions that were observed. At 18 months, children completed $71 \%$ of the target actions (approximately 21 of 29 on average) using a completely roledifferentiated strategy. By 24 months, the average number of RDBMs had increased to just over 27 out of 29 or $94 \%$ of the test battery. A repeated measures ANOVA with a Greenhouse-Geisser correction found a significant effect of age on the number of RDBMs, $F(3.595,115.054)=48.291, P<0.001$. The number of RDBMs per session appears to asymptote around 21 to 22 months. Bonferroni-corrected post hoc tests revealed significant differences in the number of RDBMs performed between all age pairs except 21 and 22 months, 21 and 23 months, 22 and 23 months, 22 and 24 months, and 23 and 24 months (all $P s<0.02$ ). There was no effect of infant handedness status (right hand preference versus no hand preference; see section 3.2) on the number of RDBMs performed as toddlers ( $P>0.05)$. There was also no effect of toddler handedness status (right hand preference versus left hand preference; see section 3.2) on the number of RDBMs $(P>0.05)$.

Data from the adult control group is reported in Table 3. The adults approached the new test battery similarly to the toddlers, performing nearly every target action with an RDBM strategy $(M \pm \mathrm{SD}=28.4 \pm 0.74)$. We calculated each adult participant's percentage of righthand use using the formula $(R / R+L) * 100$, where $\mathrm{R}$ represents the number of right-hand actions and $\mathrm{L}$ represents the number of left-hand actions. The measure captured hand use preferences along a full continuum from strongly left to exclusively right with a range of percent right-hand use from $7 \%$ to $100 \%(M \pm \mathrm{SD}=80.8 \pm 29.1)$, indicating it was capable of discriminating both degree and direction of preference.

\subsection{The relationship between infant and toddler handedness}

We first calculated each child's percentage of right-hand use (\%R) separately for every visit using the formula described previously for adults. Next, we computed $95 \%$ Confidence Intervals (CI) derived from each child's monthly right-hand use percentages for their block of infant visits (6 to 14 months) and separately for their block of toddler visits (18 to 24 months). Children were classified as left-handed if their mean $\% \mathrm{R}+\mathrm{CI}<50 \%$. Children were classified as right-handed if their mean $\% \mathrm{R}-\mathrm{CI}>50 \%$. Values that were within $5 \%$ of the $50 \%$ level were also considered lateralized. All other children were classified as having no statistically reliable preference (mean $\% \mathrm{R} \pm \mathrm{CI}$ crossed the $50 \%$ level by more than $5 \%$ ). As infants, 15 children were lateralized right (39\%), whereas the remaining 23 children had no hand preference (61\%). As toddlers, 37 of the children were lateralized (97\%) with only one child maintaining no preference. The majority of toddlers were classified as righthanded $(76 \%)$ with a larger minority of left-handers $(21 \%)$ than what has traditionally been reported for adult samples (e.g., Annett, 1985). 
We identified five patterns of handedness trajectories when comparing the groups derived from the CI calculations linking infant to toddler hand-use preferences (Table 4). A large minority of children $(N=14)$ exhibited a consistent right-hand preference across both testing blocks (Pattern 1). In this group, the percentage of right-hand use ranged from $62.3 \%$ to $81.0 \%(M \pm \mathrm{SD}=69.4 \pm 6.2 \%)$ during the infant sessions. The range for their toddler sessions was $66.2 \%$ to $95.2 \%$ right-hand use $(M \pm \mathrm{SD}=82.2 \pm 9.1 \%)$. Among children that did not exhibit a consistent hand-use preference as infants, most $(N=15)$ became righthanded as toddlers (Pattern 2). Their infant scores ranged from $41.0 \%$ to $61.2 \%(M \pm \mathrm{SD}=$ $52.4 \pm 6.1 \%)$ and toddler scores ranged from $61.6 \%$ to $98.4 \%(M \pm \mathrm{SD}=84.2 \pm 12.1 \%)$ for right-hand use. Likewise, some children with no hand-use preference as infants became lefthanded as toddlers ( $N=7$; Pattern 3$)$. Similar to their rightward developing counterparts, the infant scores for this group ranged from $42.6 \%$ to $62.1 \%(M \pm \mathrm{SD}=53.3 \pm 7.4 \%)$ right-hand use. As toddlers, the range was $21.9 \%$ to $37.9 \%$ ( $M \pm \mathrm{SD}=30.7 \pm 6.5 \%)$, reflecting the leftward shift in preferred hand use.

The remaining two trajectory patterns were each found in only one participant. One child maintained no hand-use preference between the infant and toddler sessions (Pattern 4). As an infant, the mean percentage of right-hand use was $49.5 \%$ and as a toddler it was $60.1 \%$. Interestingly, one child shifted in hand preference direction between infancy and toddlerhood (Pattern 5). As an infant, this child was mildly right-handed, with a mean of $67.6 \%$ right-hand use, but as a toddler, shifted to a strong left hand-use preference with a mean of only $19.7 \%$ right-hand use. Notably, no child exhibited a left hand-use preference during infancy in our sample and thus, no shifts from left to right preference were possible.

\section{Discussion}

The goals of the current study were to first create a battery of test items to measure hand use preferences for RDBM actions and second to connect early patterns of unimanual hand use preferences in infancy (acquisition of objects) to later patterns of bimanual hand use preferences during toddlerhood (RDBM). The number of test items, as well as the number of assessments, is unmatched in the preexisting literature characterizing the development of handedness. We found that our new battery elicited RDBM actions in toddlers 18 to 24 months of age as well as adults. Moreover, the new battery uniquely identified left- and right-handedness in both test populations. An important point regarding testing is that toddlers were capable of completing many more trials than researchers have traditionally attributed to this age period. In addition to the 29 items described in Table 1, toddlers also completed 20-25 other trials at each session unrelated to the analyses presented here (these were trials related to problem-solving, tool use and construction abilities). This underscores the need to have well-defined constructs that capture the behavior of interest in more than just a single trial, and highlights the fact that this can be done in the context of administering other measures in tandem.

A noteworthy finding from our results is that there is no single pattern in the development of handedness, an observation that serves to reconcile conflicting reports from other longitudinal efforts with smaller sample sizes regarding fluctuations in handedness and individual differences (e.g., Corbetta \& Thelen, 1999). From our analyses of the 16 visits, we identified five patterns of handedness trajectories, of which three patterns characterized $95 \%$ of our sample. These patterns were (1) children with a consistent right hand preference throughout the duration of the study; (2) children with no preference as infants that became right-handed as toddlers; and (3) children with no preference as infants that became lefthanded as toddlers. Overall, $39 \%$ of children exhibited a consistent right hand preference across all sessions from 6 to 14 months and again from 18 to 24 months. By the conclusion of the study, the number of right-handed children had grown to $76 \%$ and a further $21 \%$ had 
become left-handers, leaving just one child whom we could not yet identify as left- or righthanded.

One interpretation of these findings is that stable handedness exists in infancy. Some, but not all, infants show clear hand preferences for acquiring objects unimanually. These preferences are stable and persist into their second year of life as bimanual skills develop. Moreover, infants without a stable preference seem to become lateralized as toddlers, contradicting theories that handedness stabilizes around school age (e.g., Gesell \& Ames, 1947; McManus et al., 1988). Because of the variability that has long been associated with early hand preferences, the issue of when handedness "emerges" or becomes consolidated has been greatly debated. The current study provides some evidence that handedness might begin to stabilize earlier than traditionally assumed, but there are a few caveats. First, we caution that these findings need to be replicated. Second, we observed a child in our sample shift direction of preference from right-handedness as an infant to left-handedness as a toddler. Ramsay (1980) also reported a few cases of directional shifts in hand preference from unimanual to bimanual hand use. One possibility is that the infant handedness assessment lacks sensitivity, in particular for detecting left-handedness, although this is not likely to be the case as we have identified left-handed infants in previous cohorts with this measure. Third and related to the second point is the level of left-handedness we observed in toddlers $(21 \%)$ was much higher than that what has typically been reported in adults $(\sim 10 \%)$. Nonetheless, this finding matches previous reports of a higher incidence of lefthandedness among toddlers and preschoolers (e.g., Marschik et al., 2008; Ramsay, Campos \& Fenson, 1979; Tirosh, Stein \& Harel, 1999). Indeed, Annett (1985) reported a higher proportion of left-handers in her sample of 3.6 to 5.3 year-old children tested with a proficiency task as compared to her sample of teenaged children (13 to 15 years old). Taken together, these patterns suggest that the development of left-handedness is not well understood. In addition, it raises the question of whether the timing or the trajectory of lefthandedness differs from that of right-handedness. It is difficult to track left-handedness in studies with small samples, given that most infants, like adults, show a rightward asymmetry. The issues surrounding left-handedness are critical avenues for future research (see Previc, 1991 for additional discussion).

Interestingly, the percentage of preferred hand use increased significantly between the infant and toddler sessions. For example, among consistent right-handers, the mean percentage of right-hand use for the 6 to 14 month period was $69.4 \%$ whereas the mean percentage of right-hand use for the 18 to 24 month period was $82.2 \%$. Whether this is a difference in the manual skills sampled (unimanual versus bimanual) is also a question for future research. There is some evidence from the nonhuman primate literature to suggest that bimanual tasks elicit stronger hand-use preferences compared to unimanual tasks (e.g., Lilak \& Phillips, 2007). Of course, the nonhuman primate work is typically done with mature adults and not infants. Targeting skillful behavior is critical in measuring asymmetries in hand use, regardless of the sample population. Developmental test items should involve activities that balance challenge with a rapidly changing repertoire. As such, unimanual reaching may be appropriate for measuring hand-use preferences in infancy when reaching for and acquiring objects is a new and relatively difficult skill. As toddlers, acquiring objects has become routine and may not elicit as strong of a bias in hand use as RDBM, which is likely the new manual challenge for that age period.

The shift to more robust hand use preferences may also be a developmental phenomenon. We have hypothesized that the development of handedness is a cascade involving early postural asymmetries (e.g, Michel, 1981), subsequent object acquisition and manipulation (e.g., Hinojosa, Sheu \& Michel, 2003), and finally complex coordination between the hands. The next steps in evaluating this hypothesis are to characterize hand use preferences for 
partially differentiated bimanual actions and then examine the links between this earlier form of RDBM and preferences for reaching, unimanual manipulation, and fully differentiated RDBM. It will also be critical to conduct follow-up analyses as toddlers approach school entry to further address timing questions surrounding the development of hand use preferences. Finally, future work should explore the implications of multiple trajectories and potential differences in developmental timing, as it pertains to handedness specifically, but also how it shifts our notions of development in general as we move as a field away from a one size fits all model towards understanding individual differences and outcomes.

\section{Acknowledgments}

The authors gratefully acknowledge the families that participated in this project. The research described in this report was supported by NSF grant DLS 0718045 (GFM) and NIH/NICHD training grant T32-HD007376 (ELN).

\section{References}

Annett, M. Left, right, hand and brain: The right shift theory. Hillsdale, NJ: Lawrence Erlbaum Associates; 1985. (see page 392)

Berthier N, Keen R. Development of reaching in infancy. Experimental Brain Research. 2006; 169:507-518.

Carlson DF, Harris LJ. Development of the infant's hand preference for visually directed reaching: Preliminary report of a longitudinal study. Infant Mental Health Journal. 1985; 6:158-174.

Corbetta D, Thelen E. Lateral biases and fluctuations in infants spontaneous arm movements and reaching. Developmental Psychobiology. 1999; 34:237-255. [PubMed: 10331149]

Fagard, J. Changes in grasping skills and the emergence of bimanual coordination during the first year of life. In: Connolly, KJ., editor. The Psychobiology of the Hand. Mac Keith Press; London: 1998. p. 123-143.

Fagard J, Jacquet A. Onset of bimanual coordination and symmetry versus asymmetry of movement. Infant Behavior \& Development. 1989; 12:229-235.

Fagard J, Lockman JJ. The effect of task constraints on infants' (bi)manual strategy for grasping and exploring objects. Infant Behavior \& Development. 2005; 28:305-315.

Fagard J, Marks A. Unimanual and bimanual tasks and the assessment of handedness in toddlers. Developmental Science. 2000; 3:137-147.

Fagard J, Pezé A. Age changes in interlimb coupling and the development of bimanual coordination. Journal of Motor Behavior. 1997; 29:199-208. [PubMed: 12453779]

Fagard J, Spelke E, Hofsten C. Reaching and grasping a moving object in 6-, 8-, and 10-month-old infants: laterality and performance. Infant Behavior \& Development. 2009; 32:137-146. [PubMed: 19185922]

Ferre CL, Babik I, Michel GF. Development of infant prehension handedness: A longitudinal analysis during the 6- to 14-month age period. Infant Behavior \& Development. 2010; 33:492-502. [PubMed: 20619463]

Geerts WK, Einspieler C, Dibiasi J, Garzarolli B, Bos AF. Development of manipulative hand movements during the second year of life. Early Human Development. 2003; 75:91-103. [PubMed: 14652162]

Gesell A, Ames LB. The development of handedness. The Journal of Genetic Psychology. 1947; 70:155-175. [PubMed: 20260602]

Greaves S, Imms C, Krumlinde-Sundholm L, Dodd K, Eliasson A. Bimanual behaviours in children aged 8-18 months: A literature review to select toys that elicit the use of two hands. Research in Developmental Disabilities. 2012; 33:240-250. [PubMed: 22093670]

Hinojosa T, Sheu C, Michel GF. Infant hand-use preferences for grasping objects contributes to the development of a hand-use preference for manipulating objects. Developmental Psychobiology. 2003; 43:328-334. [PubMed: 15027416] 
Kimmerle M, Ferre CL, Kotwica KA, Michel GF. Development of role-differentiated bimanual manipulation during the infant's first year. Developmental Psychobiology. 2010; 52:168-180. [PubMed: 20127887]

Kimmerle M, Mick LA, Michel GF. Bimanual role-differentiated toy play during infancy. Infant Behavior \& Development. 1995; 18:299-307.

Lilac AL, Phillips KA. Consistency of hand preference across low-level and high-level tasks in capuchin monkeys (Cebus apella). American Journal of Primatology. 2007; 69:1-12.

Marschik PB, Einspieler C, Strohmeier A, Plienegger J, Garzarolli B, Prechtl HFR. From the reaching behavior at 5 months of age to hand preference at preschool age. Developmental Psychobiology. 2008; 50:511-518. [PubMed: 18551468]

McManus IC, Sik G, Cole DR, Mellon AF, Wong J, Kloss J. The development of handedness in children. British Journal of Developmental Psychology. 1988; 6:257-273.

Michel GF. Right handedness: A consequence of infant supine head-orientation preference? Science. 1981; 212:685-687. [PubMed: 7221558]

Michel G, Ovrut MR, Harkins DA. Hand-use preference for reaching and object manipulation in 6through 13-month-old infants. Genetic, Social and General Psychology Monographs. 1985; 111:407-427.

Michel GF, Tyler AN, Ferre C, Sheu C. The manifestation of infant hand-use preferences when reaching for objects during the seven to thirteen-month age period. Developmental Psychobiology. 2006; 48:436-443. [PubMed: 16886180]

Previc FH. A general theory concerning the prenatal origins of cerebral lateralization in humans. Psychological Review. 1991; 98:299-334. [PubMed: 1891521]

Ramsay DS. Onset of unimanual handedness in infants. Infant Behavior \& Development. 1980; 3:377385.

Ramsay DS, Campos JJ, Fenson L. Onset of bimanual handedness in infants. Infant Behavior \& Development. 1979; 2:69-76.

Ramsay DS, Weber SL. Infants' hand preference in a task involving complementary roles for the two hands. Child Development. 1986; 57:300-307.

Rochat P. Object manipulation and exploration in 2- to 5-month-old infants. Developmental Psychology. 1989; 25:871-884.

Rönnqvist L, Domellöf E. Quantitative assessment of right and left reaching movements in infants: A longitudinal study from 6 to 36 months. Developmental Psychobiology. 2006; 48:444-459. [PubMed: 16886181]

Tirosh E, Stein M, Harel J. Hand preference as related to development and behavior in infancy. Perceptual and Motor Skills. 1999; 89:371-380. [PubMed: 10597571] 


\section{Highlights}

- We tracked the development of handedness in 38 children over 16 visits.

- We introduced a new measure for assessing bimanual handedness.

- We found that some infants had stable preferences, persisting across skills.

- Other infants had no preference, but became right- or left-handed by 2 years.

- Similar to adults, the majority of children were right-handed. 


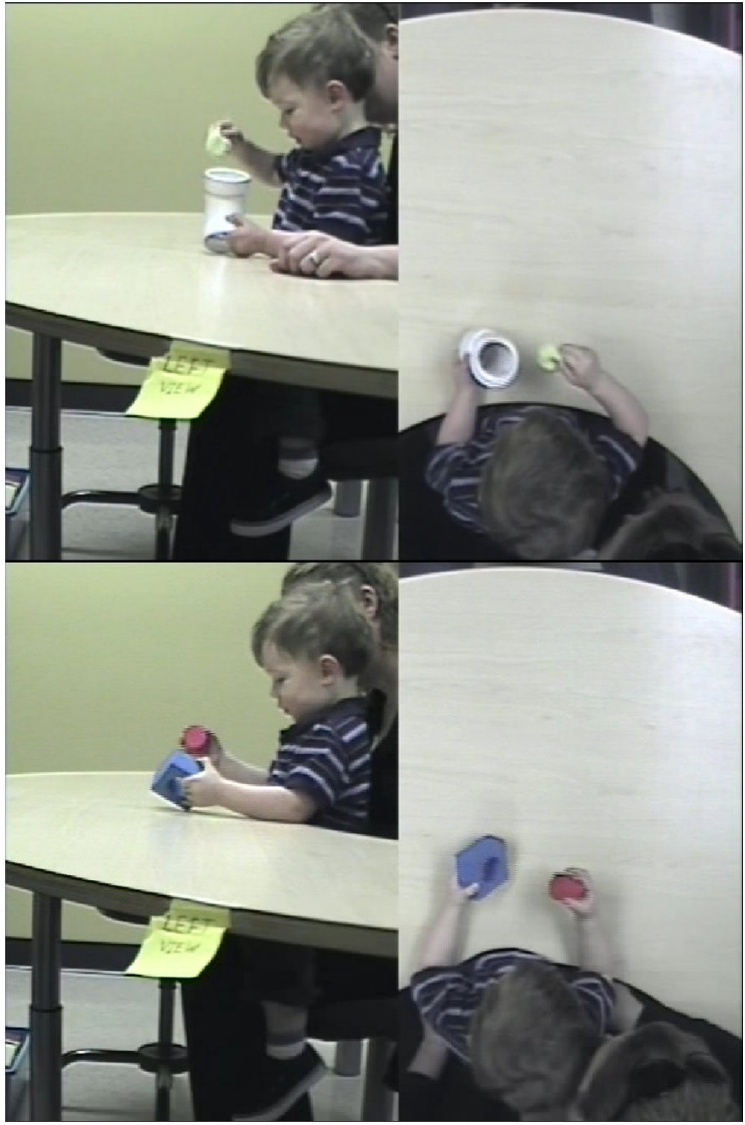

Figure 1.

Examples of RDBM actions. Top panel: Ball-in-Tube. Bottom panel: Foam-Peg-Block. One hand stabilizes the object and the other hand performs the target manipulation. 


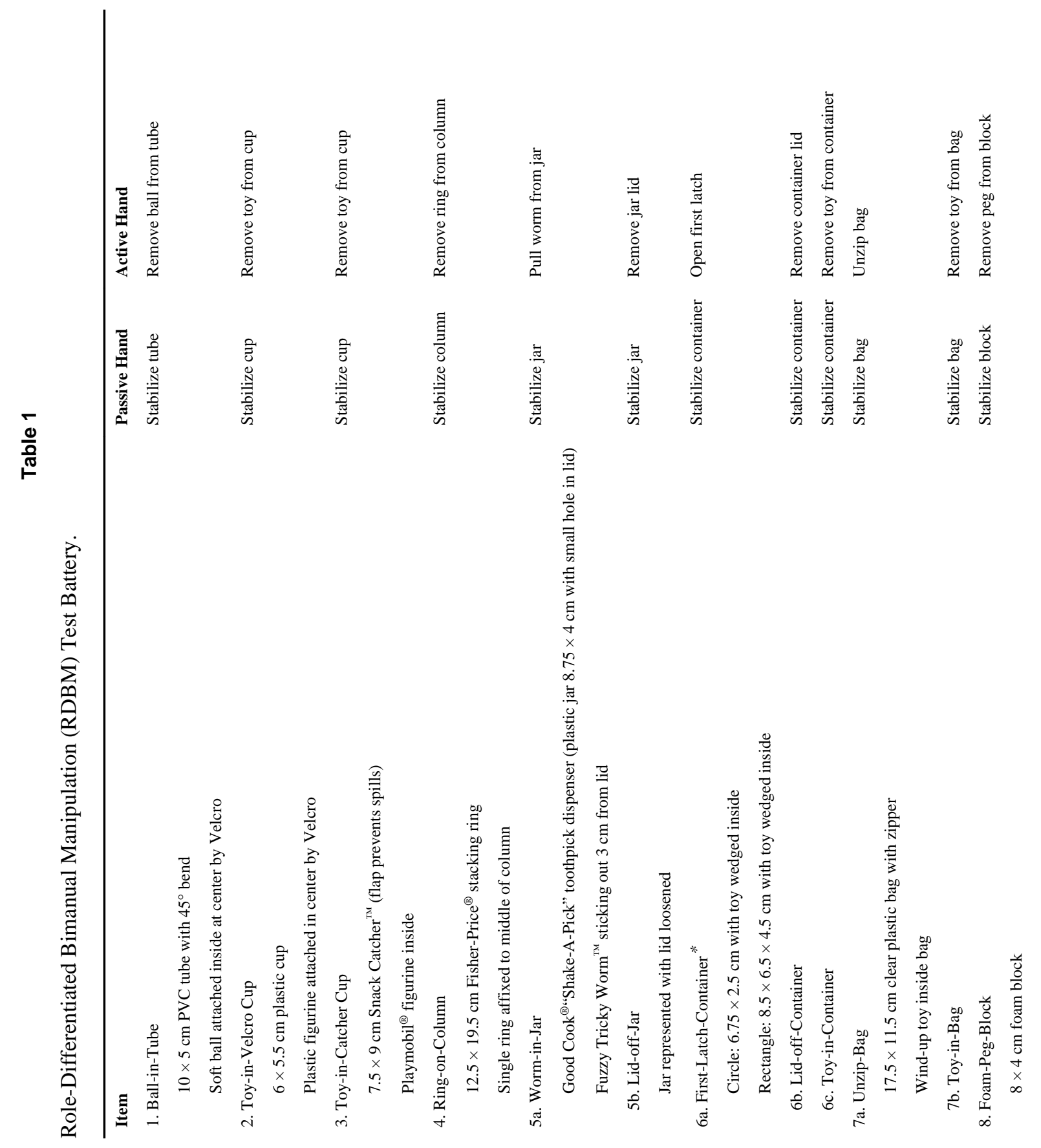

Infant Behav Dev. Author manuscript; available in PMC 2014 April 01. 


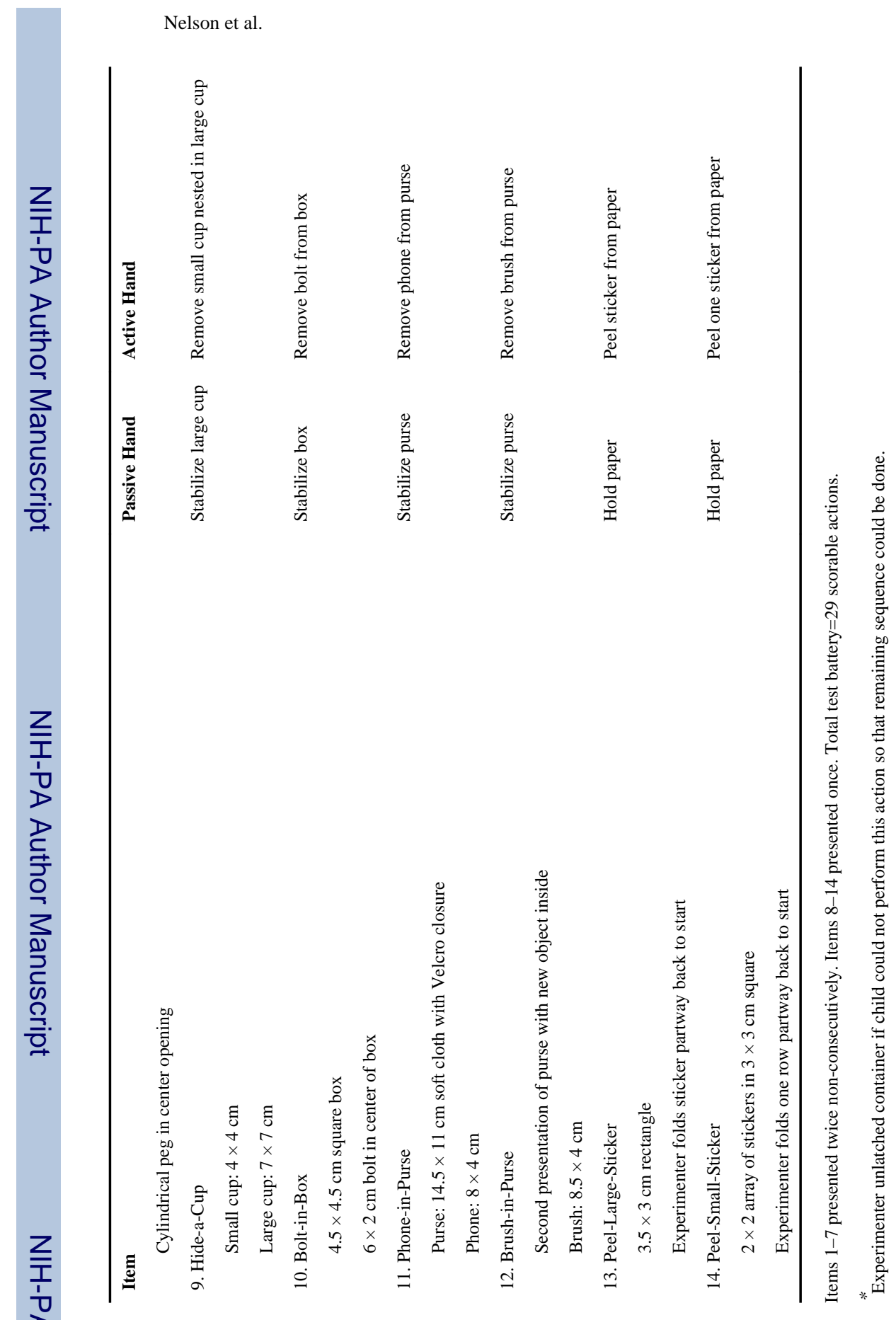

Infant Behav Dev. Author manuscript; available in PMC 2014 April 01. 


\section{Table 2}

Number of toddlers who provided data and the mean number of RDBM actions by age.

\begin{tabular}{lll}
\hline Age (mos.) & Number of toddlers & Mean RDBM actions (SD) \\
18 & 38 & $20.7(3.4)$ \\
19 & 37 & $22.7(3.3)$ \\
20 & 38 & $24.7(2.6)$ \\
21 & 38 & $26.0(2.4)$ \\
22 & 38 & $26.5(1.6)$ \\
23 & 36 & $26.8(1.7)$ \\
24 & 38 & $27.3(1.3)$ \\
\hline
\end{tabular}

RDBM=Role-Differentiated Bimanual Manipulation. $\mathrm{SD}=$ Standard Deviation. 


\section{Table 3}

Individual hand use data for adults.

\begin{tabular}{llll}
\hline ID & Index & \# RDBMs & Gender \\
1 & 6.9 & 29 & $\mathrm{~F}$ \\
2 & 25.0 & 28 & $\mathrm{M}$ \\
3 & 65.5 & 29 & $\mathrm{M}$ \\
4 & 71.4 & 28 & $\mathrm{M}$ \\
5 & 74.1 & 27 & $\mathrm{~F}$ \\
6 & 82.8 & 29 & $\mathrm{~F}$ \\
7 & 89.7 & 27 & $\mathrm{~F}$ \\
8 & 96.6 & 29 & $\mathrm{~F}$ \\
9 & 100.0 & 28 & $\mathrm{~F}$ \\
10 & 100.0 & 28 & $\mathrm{~F}$ \\
11 & 100.0 & 28 & $\mathrm{~F}$ \\
12 & 100.0 & 29 & $\mathrm{~F}$ \\
13 & 100.0 & 29 & $\mathrm{~F}$ \\
14 & 100.0 & 27 & $\mathrm{M}$ \\
15 & 100.0 & 29 & $\mathrm{~F}$ \\
\hline
\end{tabular}

Index $=$ Percentage of right-hand use. Calculated with the formula $(\mathrm{R} / \mathrm{R}+\mathrm{L}) * 100$, where $\mathrm{R}=\mathrm{Right}, \mathrm{L}=\mathrm{Left}$. RDBM=Role-Differentiated Bimanual Manipulation. $\mathrm{M}=$ Male, $\mathrm{F}=$ Female. 


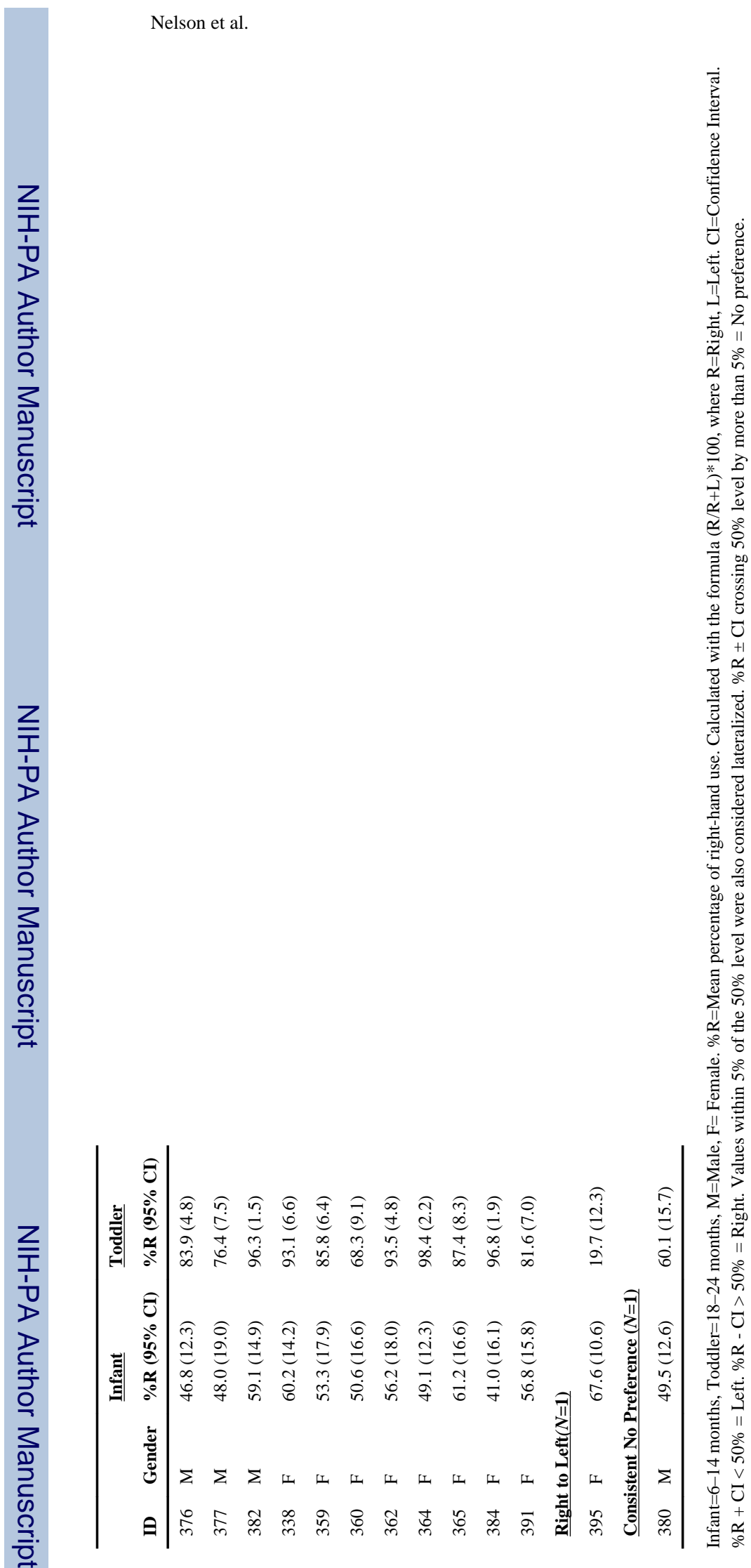

Infant Behav Dev. Author manuscript; available in PMC 2014 April 01. 